\title{
Influence De La Bancarisation Et Du Credit Bancaire Sur La Croissance Economique Dans La Zone Cemac
}

\author{
Pr Noula Armand Gilbert, Maître De Conférences \\ Bkwayep Nguemnang Y. Rodrigue, Doctorant \\ Dr Mba Fokwa Arsène
}

Faculté des Sciences Economiques et de Gestion-

Université de Dschang- Cameroun

doi: 10.19044/esj.2016.v12n31p373 URL:http://dx.doi.org/10.19044/esj.2016.v12n31p373

\begin{abstract}
The CEMAC countries have decided to develop the banking sector to ensure economic growth ${ }^{48}$ for a sustainable development, given that the banking system leads to investments. Our study aims at analyzing the influence of bank credit and banking rate on economic growth in the CEMAC zone from time series data during 1980-2014 (CD -ROM, WBI2014). The econometric analysis that we have chosen was inspired by the generalized least squares method. The model we preferred was that of Hague (2000) where the Gross Domestic Product is the dependent variable for assessing the level of economic growth while the bank credit and the banking rate are the main explanatory variables. The results indicate that the variables are significant thus, the banking rate affects economic growth positively. Following these results one could think about new strategies that will help increase the banking rate which is still very low in the Sub-region.
\end{abstract}

Keywords: Bank loans, banking rate, Economic Growth, CEMAC zone

Résumé

Le système bancaire contribuant aux investissements, les pays de la CEMAC (Communauté Economique et Monétaire de l'Afrique Centrale) ont décidé de développer ce secteur afin d'assurer une croissance économique ${ }^{49}$

\footnotetext{
${ }^{48}$ In economics, growthrefers to the annual change, expressed as a percentage of GDP (Gross Domestic Product) and GNP (Gross National Product) specifically, increasedsustainable dimension, accompanied by structural changes and leading to the improvement of living standards.

${ }^{49}$ En économie, la croissance désigne l'évolution annuelle, exprimée en pourcentage, du PIB (Produit intérieur brut) ou du PNB (Produit national brut) plus précisément,
} 
soutenue pour un développement durable. Notre étude se propose d'analyser l'influence du crédit bancaire et de la bancarisation sur la croissance économique dans la zone CEMAC à partir des données en séries temporelles durant la période 1980 à 2014 (CD -ROM, WBI-2015). L’analyse économétrique que nous avons empruntée s’est inspirée de la méthode des Moindres carrés généralisés. Le modèle retenu est celui de Haye (2000) où le Produit Intérieur Brut représente la variable expliquée qui permet d’évaluer le niveau de croissance économique tandis que le crédit bancaire et le taux de bancarisation sont les principales variables explicatives. Les résultats obtenus nous montrent que les variables retenues sont significatives et que le taux de bancarisation agit positivement sur la croissance économique. A la suite de ces résultats on pourrait penser aux stratégies nouvelles permettant d'augmenter le taux de bancarisation qui reste encore très bas dans la sousrégion.

Mots clés: Crédits bancaires, Bancarisation, Croissance économique, zone CEMAC

\section{Introduction}

L’activité bancaire est apparue avec le développement du commerce mondial aux $15^{\text {ieme }}$ et $16^{\text {ieme }}$ siècle en Europe, en Asie et en Amérique. Après avoir négocié leurs marchandises, les négociants gardaient leur monnaie en or dans les institutions financières où ils avaient la sécurité de ne pas la perdre, et cet or ne sortait que pour son déposant. Afin de faciliter les échanges les commerçants ont commencé à accepter entre eux les reçus qu'ils ont eu auprès des banques. Les banquiers ayant constaté ce nouveau moyen de payement, ont commencé à émettre des reçus sans contrepartie en or, mais sans reconnaissance de dette de la part des emprunteurs. Cette situation posait des problèmes lorsque les détenteurs se présentent au même moment pour retirer leur or. D’où l'apparition du rôle d'autorégulation du banquier qui consiste à limiter les prêts accordés et gérer la liquidité dont il dispose.

Le rôle du banquier a beaucoup évolué avec le temps et c’est ainsi qu’il assure le rôle d’intermédiaire financier entre les agents à capacité de financements et les agents à besoin de financements. Afin de satisfaire ces deux catégories d'agent, la banque détient leurs différents comptes bancaires. La bancarisation reste alors un facteur très important pour les banques et par conséquent pour la croissance économique. Au $20^{\text {ieme }}$ siècle, Schumpeter (1912) soulignait la grande importance des banques dans le

l'accroissement durable de sa dimension, accompagné de changements de structure et conduisant à l'amélioration du niveau de vie. 
fonctionnement du système économique et leur apport bénéfique à la croissance à travers le financement de l'innovation. L'Afrique subsaharienne particulièrement l'Afrique centrale sera notre échantillon auquel nous déterminerons l’impact du crédit bancaire et de la bancarisation sur la croissance économique.

L'investissement est une composante du revenu et un facteur décroissance. Il se pose alors un problème fondamental à savoir: Ia bancarisation et les crédits bancaires peuvent-ils être des facteurs importants pour la croissance économique dans la zone CEMAC?

Nous essayerons d'analyser l'importance de la bancarisation et du crédit bancaire sur la croissance économique en zone CEMAC. Car nous pensons qu'un taux de bancarisation élevé et le financement par crédit bancaire ont un impact positif sur la croissance économique en zone CEMAC.

\section{Revue de la litterature \\ Revue de la litterature}

La théorie économique, comme tous les domaines de connaissance a toujours été partagée entre plusieurs approches. Ainsi, nous pouvons observer deux pôles sur l'importance du système financier dans la croissance à travers l'histoire de la théorie économique.

D'une part, la lignée de Bagehot (1873), qui argumente que le succès du développement britannique est dû à la supériorité de son marché financier, qui avait une facilité relative à mobiliser l'épargne pour financer les divers investissements à long terme. Ainsi, les opportunités d'accès au financement des entreprises auraient été décisives pour la mise en place de nouvelles technologies en Angleterre. Par conséquent, le sousdéveloppement économique serait lié à l'impossibilité de mobilisation des ressources, caractéristique d'un système financier atrophié ou quasi inexistant. Schumpeter (1912) avance que les services financiers, en particulier les crédits bancaires sont indispensables pour la croissance économique dans la mesure où ils améliorent la productivité en encourageant l'innovation technologique. Selon l'auteur, «on ne peut devenir entreprise qu’en ayant été préalablement un débiteur». Ici, ce penseur met en relief le rôle central du crédit dans l'aspiration de la croissance économique et il indique que le banquier permet d'identifier les entrepreneurs qui ont les meilleures chances de réussir la procédure d'innovation. L'entrepreneur innovateur a besoin de moyens financiers pour mettre en œuvre de nouvelles technologies de production, et le rôle du banquier est primordial dans le choix de ces nouvelles technologies. On peut conclure donc que le développement financier stimule la croissance à travers l'allocation efficace des ressources. 
D'autre part, Robinson (1952) et Lucas (1988) auparavant ne croyaient pas à l'importance de la relation entre finance et croissance économique. Malgré les divergences de points de vue sur la question, un lien positif se dégage de la majorité des travaux entre le volume du financement bancaire et la création de richesse d'une nation. Aussi, le rapport crédit bancaire à l'économie sur le Produit Intérieur Brut (PIB) est, selon certaines études, un très bon indicateur. Poursuivant la même logique, une abondante littérature théorique et empirique détermine un lien positif entre le développement financier et la croissance économique connue comme l'accroissement du PIB annuel global d'une nation. McKinnon et Shaw (1973) montrent dans leurs travaux que le secteur financier joue un rôle très important dans le processus de croissance économique.

Cependant, à travers leur école de répression financière qui se caractérise par la fixation des taux d'intérêt réels en dessous de leur niveau d'équilibre de marché (taux d'intérêt plafonnés), des montants élevés des réserves bancaires, une politique d'allocation sélective du crédit qui conduit à des investissements intensifs en capital et de piètre qualité, l'obligation faite aux banques de financer prioritairement les projets gouvernementaux peu rentables. Elle est basée sur la théorie de la croissance de la préférence pour la liquidité développée par Keynes (1936), qui avance que pour assurer le plein emploi, le niveau d'équilibre du taux d'intérêt doit être inférieur au taux de préférence de la liquidité. Ainsi les taux d'intérêts doivent être le plus faible possible afin d'éviter la chute des revenus. De plus, la répression financière va de la mainmise de l'Etat sur le système financier à la pure nationalisation des banques.

Cette situation d'oppression financière ne peut que générer selon ces deux auteurs, de l'inflation couplée avec un ralentissement de la croissance économique. Ainsi, la libéralisation financière entraîne un développement financier conduisant à une meilleure mobilisation de l'épargne et sa répartition efficiente pour le financement des investissements.

Cette façon d'appréhender le problème sera simplifiée par les travaux de certains penseurs notamment, Kapur (1976), Collier \& Gunning (1999) qui considèrent que le développement financier est synonyme d'assouplissement des conditions de taux d'intérêt et de proximité des marchés financiers. En outre, Bencivenga et Smith (1991) montrent à travers leurs travaux qu'il existe un lien positif entre le financement bancaire et la croissance économique. En fait, sur le plan empirique, nombreux sont les économistes qui ont étudié la nature du lien entre financement bancaire et croissance économique notamment Meagon (2005) a étudié l'impact du financement bancaire sur la croissance économique au Sénégal. Il ressort de son analyse que la croissance au Sénégal s’explique principalement par le niveau du crédit à l'économie et les dépôts totaux. Soumare (2009) a analysé 
le même problème sur l'économie malienne et a abouti à la conclusion selon laquelle le crédit bancaire agit négativement sur la croissance et les dépôts agissent positivement mais faiblement sur elle.

Haye (2000) a étudié un échantillon de 12 pays donc six développés et six autres en développement et l'auteur conclut que le crédit bancaire influence négativement la croissance des pays en voie de développement, mais positivement et significativement sur les pays développés ; Dembélé (2010) analyse la contribution du crédit bancaire sur la croissance économique ivoirienne à partir d'un modèle à équation simultanée l'auteur trouve par la méthode des triples moindres carrés que le crédit à l'économie agit positivement et significativement sur la croissance économique et Ngono (2009) montre à partir d'une méthode de régression multiple pas à pas descendant (stepwise) dans le cas du financement bancaire sur l'évolution du Pib au Cameroun que le crédit au secteur privé n’influence pas à court et à long termes le Pib réel.

\section{Methodologie}

Les données collectées dans le cadre de ce travail sont de source secondaire, en provenance de la publication annuelle de la Banque Mondiale, plus précisément dans «le livre des indicateurs mondiaux de développement» contenu dans le CD -ROM (WBI-2015).L'étude couvre la période allant de 1980 à 2014. Ce choix se justifie par le souci d'intégrer les diverses évolutions qu'ont connues la politique du crédit et de la bancarisation au sein de la sous-région notamment, la zone CEMAC.

Notre travail sera organisé en trois étapes dont la première est consacrée à la présentation des variables, la deuxième à la présentation du modèle et enfin les tests statistiques à la troisième.

\section{Variable expliquee}

Taux de croissance Yi,t: PIB par habitant, prix de1980-2014. A partir de cette variable nous calculons la variable expliquée, à savoir le taux de croissance réel par habitant, en soustrayant le PIB à la période t-1 au PIB de la période $\mathrm{t}$.

\section{Variables explicatives}

$\boldsymbol{X i}, \boldsymbol{t}$ :Matrice des variables servant de noyau dans une étude des déterminants de la croissance. Les variables sont les suivantes :

- INV: ratio (FBCF + variations de stocks)/ PIB. L'investissement est une variable clef de la croissance et devrait avoir un fort effet positif parce-que l'investissement crée l'emploi et par conséquence la croissance.

- INF: Taux d'inflation. Ce qui justifie l'introduction du taux d'inflation comme variable explicative de la croissance est le concept de la répression 
financière. En effet, un taux d'inflation élevé caractérise les économies où la répression financière est forte, afin que le taux d'intérêt réel soit négatif, réduisant ainsi le poids de la dette gouvernementale. Or, une forte inflation défavorise les investissements à long terme et exerce un effet nuisible à la croissance. Le signe attendu pour cette variable est donc négatif. - OUV : à l'instar de Berthelemy et Varoudakis (1998) nous utilisons le coefficient d'ouverture commerciale et nous calculons en faisant le ratio (Exportations + Importations) / PIB. Cependant, cet indicateur n'est pas optimal puisqu'en plus des orientations de la politique économique, il reflète l'influence des différences naturelles telles que la taille et l'emplacement géographique de chaque pays.

- POP : cette variable correspond, comme dans Mankiw et Weil (1992), à la somme du taux de croissance de la population ${ }^{50}$ (n), du taux de croissance du progrès technique (g) et du taux de dépréciation du capital (d) (avec g $+\mathrm{d}=0,05$ ).

- Zi,t:matrice de variables caractérisant les banques de chaque pays.

- DEP:c'est une variable du passif bancaire. Son mode de calcul correspond au ratio (Total des dépôts à vue et à terme détenus par les banques) / PIB. Elle reflète le niveau de développement et la taille du système bancaire. Selon la théorie de la croissance endogène, il existe une relation positive entre la taille des banques et la croissance.

- MASS: mesure la part des actifs assimilables à des moyens de paiement dans le PIB. C'est donc le rapport M2/PIB, qui reflète la liquidité de l'économie. Son signe attendu devrait être positif.

- CI : nous avons précisé dans ce qui précède que l'activité du crédit occupe une place centrale dans l'actif des banques et joue un rôle essentiel dans l'économie. Ainsi, pour la mesurer nous prenons le rapport du Crédit intérieur/PIB.

- LIQ : Cette variable correspond au rapport du crédit intérieur sur les dépôts. Elle reflète la liquidité des banques et donc leur capacité à faire face aux éventuels retraits de dépôts. De même, elle prend en compte l'interaction entre le passif et l'actif des banques. Son signe attendu devrait être positif.

\section{Presentation du modele}

Un grand nombre d'études analysant l'effet du système financier sur la croissance effectuent leurs analyses économétriques en coupe instantanée. L'économétrie repose ainsi sur des moyennes, ce qui suppose un

\footnotetext{
${ }^{50}$ Nous précisons que dans l'article de MankiwRomer et Weil (1992) «n » correspondent au taux de croissance de la population active. Or les données statistiques de cette variable étant difficile à obtenir pour une analyse en données de panel, nous prenons à la place le taux de croissance de la population.
} 
comportement homogène dans le temps des différentes variables. Or, les bases de données concernant l'étude de la croissance économique à long terme comportent une dimension individuelle et temporelle. L'analyse transversale n'utilise que la dimension individuelle. C'est pourquoi, les économistes étudiant les déterminants à long terme de la croissance économique ont cherché à optimiser les méthodes d'estimation afin de prendre en compte le caractère bidimensionnel de la base de données. En se basant sur le modèle de Mankiw et Weil (1992) dérivé du modèle d'Islam (1995) et Haye (2000) qui proposent d'utiliser les méthodes économétriques des données de panel pour procéder à l'estimation de l'équation de croissance, cette équation servira de base à notre démarche économétrique.

Les études qui ont tenté d'utiliser les données de panel pour analyser l'effet de la variable de bancarisation et des variables bancaires sur la croissance sont rares. A notre connaissance Berthelemy et Varoudakis (1998) utilisent cette méthode pour déterminer l'effet du système financier sur la croissance. Ces auteurs ne prennent en compte qu'une variable faisant référence à l'activité bancaire, à savoir la monnaie et laquasi monnaie $\left(\mathrm{M}_{2}{ }^{51}\right)$. Nous utiliserons cette variable dans le cadre de notre étude en ajustant une autre variable relative à l'activité des banques qui est le taux de marge bancaire.Le modèle de base ci-dessous que nous cherchons à estimer pour étudier l'impact de la bancarisation et des crédits bancaires sur la croissance économique est emprunté à Haye (2000), lequel a été adapté au contexte du Mali par Soumaré (2009), et adapté aussi dans le cas de la Côte d'Ivoire par Dembele (2010).

$$
Y_{i, t}=a X_{i, t}+b Z_{i, t}+Y_{i, t-1}+\varepsilon_{i}+\gamma_{t}+\delta_{i, t}
$$

$\boldsymbol{\varepsilon} \boldsymbol{i}$ : Effet spécifique permettant de contrôler les différences non observables qui existent entre les unités statistiques.

$\gamma t$ : Effet temporel permettant de contrôler les chocs conjoncturels qui frappent les économies.

$\boldsymbol{\delta} \boldsymbol{i}, \boldsymbol{t}$ : Perturbation aléatoire qui suit une distribution de la loi normale $\left(0, \mathrm{~s}^{2}\right)$.

Ce modèle doit être utilisé en introduisant le taux de marge bancaire pour mesurer son effet sur la croissance. Son effet attendu est inverse à celui du taux de bancarisation.

\section{Procedure d'estimation}

Lorsque les variables sont intégrées d'ordre zéro, on estime le modèle par la méthode des Moindres carrés généralisés. Par contre, si les variables sont intégrées d'ordre un, l'estimation suit la procédure du modèle à

\footnotetext{
${ }^{51} \mathrm{M}_{2}=\mathrm{M}_{1}+$ livrets d'épargne (compte épargne logement, livret de développement durable) et $\mathrm{M}_{1}=$ billets et pièces + dépôt à vue
} 
correction d'erreur de Engel et Granger ou de Johansen. Si les variables ont différent ordre d’intégration, on utilise un modèle à retard échelonné.

Lorsque les erreurs du modèle de base ne respectent pas les hypothèses classiques, notamment lorsqu'elles sont autocorrélées et hétéroscédastiques, les estimateurs des coefficients à partir de la méthode des moindres carrés ordinaires ne sont pas BLUE ${ }^{52}$.

C'est pourquoi en présence d'une autocorrélation et/ou d'une hétéroscédasticité des erreurs, il faut recourir à une méthode d'estimation qui est celle des moindres carrés généralisés(MCG)

Soit le modèle linéaire générale suivant :

$$
Y_{(T, 1)}=X_{(T, k)} a_{(K, 1)}+\varepsilon_{(T, 1)}
$$

Où $E\left(\varepsilon \varepsilon^{\prime}\right)=\omega_{\varepsilon} \neq \sigma_{t}^{2} * I\left(\Omega_{\varepsilon}\right.$ est de dimension n,n).

Nous désirons déterminer un estimateur de a qui ait les mêmes propriétés que l'estimateur des moindres carrés ordinaires (MCO) : sans biais, linéaire en Y et à variance minimale.Il est démontréque cet estimateur est donné par :

$\hat{\mathrm{a}}=\left(X^{\prime} \Omega_{\varepsilon}^{-1} X\right)^{-1}\left(X^{\prime} \Omega_{t}^{-1} Y\right)$ avec $\left.\Omega_{\varepsilon}=X^{\prime} \Omega_{\varepsilon}^{-1} X\right)^{-1}$

Cet estimateur est appelé estimateur des moindres carrés généralisés(MCG) ou encore estimateur de Aitken ${ }^{53}$.

\section{Resultats et discussions}

Après avoir présenté notre méthodologie, nous allons de ce pas présenter nos résultats, que nous interpréterons par la suite.

\section{Test de stationnarite}

Etant donné que nous travaillons sur des séries temporelles, il est nécessaire d'analyser la stationnarité ${ }^{54}$ de celles-ci. Si la série initiale n’est pas stationnaire, il faudra vérifier cette condition pour sa différence première et éventuellement, pour la différence seconde. Les prévisions économétriques fiables ne peuvent être faites que sur des séries stationnaires.

\footnotetext{
${ }^{52}$ Best Linear Unbiased Estimator

${ }^{53}$ Remarque : Lorsque les hypothèses classiques sont satisfaites, nous retrouvons l'estimateur des moindres carrés ordinaires (MCO) :â= $\left(X^{\prime} \Omega_{\varepsilon}^{-1} X\right)^{-1}\left(X^{\prime} \Omega_{\varepsilon}^{-1} Y\right)$ $\left.=X^{\prime}\left(\frac{1}{\sigma_{t}^{2}} I\right) X\right)^{-1}\left(X^{\prime}\left(\frac{1}{\sigma_{t}^{2}} \mathrm{I}\right) \mathrm{Y}\right)=\left(X^{\prime} X\right)^{-1}\left(X^{\prime} Y\right)$.

${ }^{54}$ Une série est stationnaire si elle ne comporte ni tendance, ni saisonnalité et plus généralement, aucun facteur évoluant avec le temps. Mathématiquement, on dira qu'un processus stochastique Xt est stationnaire si :

- $\mathrm{E}(\mathrm{Xt})=\mathrm{E}(\mathrm{Xt}+\mathrm{h})=\mu$ quels que soient $\mathrm{t}$ et $\mathrm{h}$, la moyenne est constante et indépendante du temps;

-la Variance est finie et indépendante du temps;

-la Covariance est indépendante du temps.
} 
Afin de savoir s’il y’a stationnarité ou pas nous procédons à une comparaison par deux méthodes à savoir :

1. Si la statistique calculée est supérieure à la statistique lue en valeur absolue, on conclut que la variable est significative ;

2. Si la probabilité est inférieure au seuil de signification choisi (5\% dans notre cas) la variable est stationnaire.

Tableau 5: Tableau de test de stationnarité

\begin{tabular}{|c|c|c|c|c|c|}
\hline \multirow[t]{2}{*}{ Variables } & \multirow[t]{2}{*}{ Probabilité } & \multirow[t]{2}{*}{$\begin{array}{l}\text { Statistique } \\
\text { calculée }\end{array}$} & \multirow{2}{*}{$\begin{array}{l}\text { Valeur } \\
\text { critique } \\
\text { à } 5 \%\end{array}$} & Stationnarité & \multirow[t]{2}{*}{$\begin{array}{c}\text { Ordre } \\
\text { d'intégration }\end{array}$} \\
\hline & & & & Oui/non & \\
\hline $\mathrm{Y}$ & 0.0000 & -6.046197 & -2.877186 & oui & $\mathrm{I}(0)$ \\
\hline$Y(-1)$ & 0.0000 & -6.961233 & -2.877274 & oui & $\mathrm{I}(0)$ \\
\hline CI & 0.0000 & -9.384823 & -2.878212 & oui & $\mathrm{I}(0)$ \\
\hline DEP & 0.0000 & -11.64953 & -2.877919 & oui & $\mathrm{I}(0)$ \\
\hline INF & 0.0000 & -12.21675 & -2.877012 & oui & $\mathrm{I}(0)$ \\
\hline INV & 0.0000 & -8.024886 & -2.877012 & oui & $\mathrm{I}(0)$ \\
\hline LIQ & 0.0000 & -7.004854 & -2.877186 & oui & $\mathrm{I}(0)$ \\
\hline MASS & 0.0000 & -9.399043 & -2.877363 & oui & $\mathrm{I}(0)$ \\
\hline MTB & 0.0002 & -4.545115 & -2.877012 & oui & $\mathrm{I}(0)$ \\
\hline OUV & 0.0000 & -13.45312 & -2.877012 & oui & $\mathrm{I}(0)$ \\
\hline POP & 0.0000 & -9.685866 & -2.878311 & oui & $\mathrm{I}(0)$ \\
\hline TB & 0.0002 & -4.545115 & -2.877012 & oui & $\mathrm{I}(0)$ \\
\hline
\end{tabular}

D’après le tableau ci-dessus nous constatons que la statistique calculée est supérieure à la statistique lue, le test de DICKEY-FULLER Augmenté montre que toutes les variables sont stationnaires au seuil de 5\%. Nous observons que toutes nos variables sont stationnaires à niveau, et au seuil de 5\%.Ceci étant,la méthode la mieux appropriée pour l'estimation de nos paramètres est celle des moindres carrés généralisés. Compte tenu du fait que nous sommes en présence d'un modèle dynamique.

\section{Iv.2 Estimation De l'effet Du Credit Bancaire Et De La Bancarisation Sur La Croissance Economique}

Les résultats obtenus seront lus à un seuil de signification de $5 \%$. La règle de décision découlera du fait que si la probabilité est inférieure à 5\%, on conclut que le coefficient de notre variable est significatif. 
Tableau 6 : Estimation des variables

\begin{tabular}{|c|c|c|c|c|}
\hline Variables & Coefficients & Std. Error & t-Statistic & Prob. \\
\hline $\mathrm{C}$ & 1020280. & 7325.698 & 139.2742 & 0.0000 \\
\hline TB & 5883.917 & 186.9909 & 31.46632 & 0.0000 \\
\hline POP & $-5.24 \mathrm{E}+09$ & 74091584 & -70.67036 & 0.0000 \\
\hline OUV & 20194.13 & 973.5597 & 20.74257 & 0.0000 \\
\hline MASS & $1.99 \mathrm{E}+08$ & 61291952 & 3.252161 & 0.0014 \\
\hline LIQ & -61391282 & 36667583 & -1.674266 & 0.0959 \\
\hline INV & $3.05 E+08$ & 26754922 & 11.41464 & 0.0000 \\
\hline INF & $-1.26 \mathrm{E}+08$ & 16817880 & -7.496006 & 0.0000 \\
\hline DEP & $2.33 \mathrm{E}+08$ & $1.48 \mathrm{E}+08$ & 1.571848 & 0.1178 \\
\hline $\mathrm{CI}$ & $-6.34 \mathrm{E}+08$ & $2.01 \mathrm{E}+08$ & -3.157016 & 0.0019 \\
\hline $\mathrm{Y}(-1)$ & 0.043838 & 0.004860 & 9.020587 & 0.0000 \\
\hline $\mathrm{AR}(1)$ & 0.685086 & 0.010239 & 66.90684 & 0.0000 \\
\hline R-squared & 0.985466 & $\begin{array}{l}\text { Meandependent } \\
\text { var }\end{array}$ & 472562.7 & \\
\hline $\begin{array}{c}\text { Adjusted R- } \\
\text { squared }\end{array}$ & 0.984537 & S.D. dependent var & 73031.12 & \\
\hline S.E. of regression & 9081.565 & Sumsquaredresid & $1.42 \mathrm{E}+10$ & \\
\hline $\begin{array}{c}\text { Durbin-Watson } \\
\text { stat }\end{array}$ & 1.675062 & F-statistic & 12.7553 & \\
\hline Inverted AR Roots & .69 & & & \\
\hline
\end{tabular}

D’après le tableau ci-dessus nous observons que la probabilité correspondant à la variable du Taux de bancarisation (TB) est inférieur à $5 \%$, donc la variable (TB) est significative et le signe de son coefficient est positif ce qui correspond au signe attendu. Donc il existe une influence positive du taux de bancarisation sur la croissance économique. Cette relation positive s'explique par le fait que si une population est bancarisée à grande échelle, la population aura plus accès aux crédits bancaires et les investissements augmenteront et réciproquement ces investissements pourront avoir plus d'effets sur la croissance économique.

La probabilité de la variable POP est inférieure à $5 \%$, donc la variable (POP) est significative comme attendu et le signe de son coefficient $-5.24 \mathrm{E}+09$ est négatif. Ceci peut s'expliquer par le fait que le taux d'investissement n'est pas proportionnel au taux de croissance de la population, on peut penser que les ressources ne sont pas bien redistribuées au niveau de la population.

La probabilité de OUV $<5 \%$, donc le coefficient de cette variable est significatif et son signe est positif comme attendu. Ceci s'explique par le fait que l'ouverture commerciale augmente la croissance économique à travers les échanges avec le Reste du Monde.

La probabilité de MASS $<5 \%$, ce qui implique que le coefficient de cette variable est significatif et son signe est positif comme attendu. Ceci 
s’explique par le fait que la croissance économique d’un pays est fonction de sa source de financement et pour entretenir et accroitre cette économie, l'Etat doit davantage investir (Dembélé, 2010).

La probabilité de LIQ>5\%, et le coefficient de cette variable n’est pas significatif. Cependant, le signe négatif peut s’expliquer par le fait que la qualité des crédits accordés (crédits à court terme et crédits à long terme) peut avoir des effets négatifs sur la qualité des investissements qu'un entrepreneur souhaite engager.

La probabilité d'INV $<5 \%$, le coefficient de cette variable est significatif, et son signe est positif comme attendu. Cecipeut se justifier par le fait que l'investissement est l'un des moteurs incontournables de la croissance économique.

La probabilité d’INF $<5 \%$, le coefficient de cette variable est significatif, et son signe est négatif comme attendu. Ce qui conforte la théorie économique.

La probabilité de DEP $>5 \%$, le coefficient de cette variable n'est pas significatif et le signe de son coefficient est positif comme attendu car, les projets de grande envergure et la taille du système bancaire permettent d'allouer des crédits aux investisseurs et par ricochet ceci entraine un effet important sur la croissance économique.

La probabilité de CI $<5 \%$, le coefficient de cette variable est significatif et le signe de son coefficient est négatif.On peut penser ici que les demandes des investisseurs ne sont que partiellement satisfaites.

\section{Tests de validation}

Les résultats des tests statistiques du modèle à un seuil de $5 \%$ nous montre que :

Pour ce qui est du test de Student, compte tenu du nombre d'observations $(\mathrm{n}=180)$ on peut comparer les valeurs calculées (t-statistic) de notre tableau à celle de la valeur théorique lue dans la table de t de Student (1.96) au seuil de $5 \%$.

Le coefficient de détermination $\mathrm{R}^{2}$ est de 0.9854 ce qui est très significatif, et par conséquent explique la variation du niveau du PIB réel à 98.54\%. Cette situation est satisfaisante pour l'explication du phénomène. Ceci est d’avantage expliqué par le coefficient de détermination corrigé $\left(\overline{R^{2}}\right)$ qui explique à $98.43 \%$ le modèle de régression. Cela nous montre que le modèle est globalement bon.

La proposition de la statistique de Durbin-Wastonempirique (DW=1.67) tend vers 2 ce qui indique l'absence d'autocorrélation des résidus. Nous vérifierons d'avantage la véracité de cette information par le test de Breusch-Godfrey.

\section{a) Test de Breusch et Godfrey}


Tableau 7: Résultats du test de Breusch et Godfrey

Breusch-Godfrey Serial Correlation LM Test

F-statistic

1.833384

3.884941

Prob. Chi-Square(2)

0.143349

Le tableau ci-dessus nous montre que la probabilité de F-statistic est supérieure au seuil de signification de $5 \%$ ce qui vient confirmer l'information selon laquelle l'autocorrélation a été corrigée lors de l'estimation des paramètres de notre modèle.

\section{b) Test d'hétéroscédasticité des erreurs : Test D'ARCH}

Le test d'ARCH ${ }^{55}$ permet de vérifier une homoscédasticité éventuelle des erreurs. Pour mener ce test, on a deux possibilités : soit effectuer un test de Fisher classique de nullité des coefficients $\rho_{\mathrm{i}}$; soit recourir à la statistique du multiplicateur de Lagrange LM qui est distribuée comme un Chi-deux $\left(\chi^{2}\right)$ à $\mathrm{p}$ degrés de liberté. Si $\mathrm{n} \times \mathrm{R}^{2}>\chi^{2}(\mathrm{p})$ lu dans la table au seuil $\alpha$, on rejette l'hypothèse nulle $\left(\mathrm{H}_{0}\right)$.On peut également comparer la probabilité au seuil $\alpha$.

Tableau 8: Résultat du test d’hétéroscédasticité

\begin{tabular}{|c|c|c|c|}
\hline \multicolumn{4}{|c|}{ Heteroskedasticity Test: ARCH } \\
\hline F-statistic & 0.023093 & Prob. F(1,183) & 0.8794 \\
\hline Obs*R-squared & 0.023343 & Prob. Chi- & 0.8786 \\
\hline
\end{tabular}

Les résultats du test révèlent que les erreurs ne sont pas hétéroscédastiques car la probabilité est bien supérieure à $5 \%$ d'où l'existence d'homoscédasticité des erreurs.

\section{Test de significativité globale du model}

La valeur obtenue de Fisher calculée est de 12.7553 ce qui est supérieure à 3.96 qui correspond à la valeur lue sur la table de Fisher au seuil de 5\%. On peut conclure que le modèle est globalement significatif.

\section{Conclusion}

L'esprit qui a prévalu le long de ces travaux était de montrer le rôle de la bancarisation et du crédit bancaire sur la croissance économique dans la zone CEMAC. Nous avons parcouru la revue de la littérature sur la bancarisation et le rôle joué par le crédit bancaire sur la croissance économique dans la zone CEMAC. Pour vérifier nos hypothèses nous avons collecté les données qui sont de source secondaire, en provenance de la publication annuelle de la Banque Mondiale, plus précisément dans «le livre des indicateurs mondiaux de développement» et nous avons fait une étude économétrique qui a consisté à tester un modèle à partir du logiciel E-

\footnotetext{
${ }^{55}$ Auto Regressive Conditional Heteroscedasticity
} 
VIEWS. Le modèle testé est un modèle dynamique qui a subi un test de stationnarité pour voir la significativité des variables. Il en découle que toutes les variables sont significatives en dehors de la variable de liquidité bancaire (LIQ) et de la variable du niveau de développement bancaire (DEP). Nous avons estimé le modèle par les moindres carrés généralisés et la significativité des variables a été de 5\%. Avec un coefficient de détermination proche de 1 , ceci nous montre que les variables explicatives retenues sont toutes significatives et que le modèle est globalement bon. Par ailleurs, les résultats nous montrent que le taux de bancarisation a un effet positif sur la croissance. Les autorités Etatiques devraient mettre sur pieds une planification bancaire qui consistera à trouver les moyens qui permettront à la population d'avoir un compte bancaire et d'avoir facilement accès aux services bancaires tout en orientant ces crédits dans les secteurs productifs tels l'agriculture, les nouvelles technologie. Au regard de quelques problèmes rencontré lors de notre travail, nous pouvons porter une nouvelle orientation sur chaque pays avec des données considérées individuellement. Car, les spécificités politiques des différents pays pourraient agir plus ou moins sur la croissance économique des pays de la zone CEMAC.

\section{References:}

1. Bagehot W. (1873): «Lombard Street: A Description of the Money Market», London: Henry.

2. Banque mondiale, World Development Indicator (2007).

3. BEAC, Etudes et Statistiques, (2006) n 301, juil-sept.

4. BEAC, Statuts (2010):Documentconsultés, www.izf.net/upload/document:Institution ns/Statutbeac, Janvier.

5. Bencivenca B.R et Smith B.D. (1991): " Financial intermediation and endogenous growth », Review of Economic Studies, vol. 58, n², pp. 195-209.

6. BerthelemyJ.etVaroudakisA.(1998): «Développementfinancier,réfor mes

financièresetcroissance:Uneapprocheendonnéesdepanel»,RevueEcon omique,vol.49, n²1,Janvier,pp.195-206.

7. COBAC - Commission Bancaire de l'Afrique Centrale, Rapport annuel (2010).

8. Collier et Gunning (1999): «Explaing African Economic Performance», journal of Economic Literature, March, Vol. 37, pp. 64-111. 
9. Dembele E. (2010) : «Contribution du financement bancaire à la croissance économique en Côte d'Ivoire ». Programme de Formation en Gestion de la Politique Economique.

10. Durbin J. et Watson G.S. (1951):Testing for serial correlation in least-squares regression, Biometrika, Vol. 37 et Vol.38.

11. Engle, R.F. and Granger, C.W.J. (1987): «Co-Integration and Error Correction: Representation, Estimation and Testing». In Econometrica, Vol. 55, pp. 251-276.

12. Haye M. (2000) : «Banques et croissance : Examen critique et analyse en données de panel» Papier présenté aux 17èmes Journées Internationales d'Economie Monétaire et bancaire LISBONNE, 7, 8, et 9 juin 2000 .

13. Hugon P. (2007) : «Rentabilité du secteur bancaire et défaillances du financement du développement. Le cas de la CEMAC », Revue Tiers Monde, Oct.-Déc., pp. 771-788.

14. Islam N. (1995): " Growth empirics: A panel data approach », Quarterly Journal of Economics, vol. CX, n4,Novembre, pp. 11271170.

15. Kapur B. (1976): "Alternative Stabilization Policies for LessDeveloped Economies", Journal of Political Economy, Vol. 84, N4, pp. 777-795.

16. Keynes J.M. (1936) : The general Theory of Employment Interest and Money, Vol. 7 The collected writings of J. M Keynes, edited by D.E.,Moggrige (London MacMillan).

17. Lucas, R. E. (1988): «On the Mechanics of Economic development», Journal of Monetary Economics, Vol. 22, $n^{\circ} 1$, pp. 342.

18. Mankiw G. Romer D. et Weil (1992): «A contribution to the empirics of economic growth », Quarterly Journal of Economics, vol. CVII, n², Mai, pp. 407-437.

19. McKinnon R. I. et Shaw (1973): « Money and Capital in economic development », Thebrookinginstitution, Washington D.C.

20. Meagon.N.R. (2005): «Money ant capital in economic development» the Broking institution Washington D.C.

21. Ngono N.A. (2003) : « Intermédiation bancaire et croissance économique au Cameroun » Maîtrise en économie monétaire et bancaire, Traductions.

22. Robinson J. (1952): " The rate of interest and other essays», London, MacMillan.

23. Schumpeter J. (1912): «The theory of Economic Development» Cambridge, MA: Harvard University Press. Servicesacross countries, World Bank, 52p. 
24. Soumaré S. (2009) : «Contribution du financement bancaire à la croissance économique en Afrique Subsaharienne : l'expérience Malienne » Programme de Formation en Gestion de la Politique Economique (Abidjan). 